\title{
Analysis of oncological safety of autologous fat grafting after immediate breast reconstruction
}

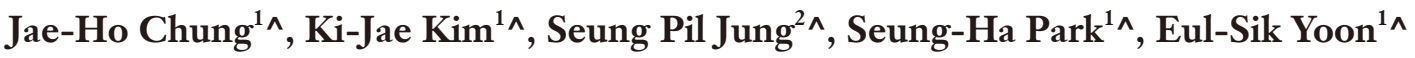 \\ ${ }^{1}$ Department of Plastic and Reconstructive Surgery, Korea University Hospital, Seoul, Republic of Korea; ${ }^{2}$ Division of Breast and Endocrine Surgery, \\ Korea University Hospital, Seoul, Republic of Korea \\ Contributions: (I) Study concept and design: JH Chung, ES Yoon; (II) Administrative support: None; (III) Provision of study materials or patients: \\ None; (IV) Collection and assembly of data: KJ Kim, JH Chung; (V) Data analysis and interpretation: JH Chung, KJ Kim; (VI) Manuscript writing: \\ All authors; (VII) Final approval of manuscript: All authors. \\ Correspondence to: Eul-Sik Yoon, MD, PhD. Department of Plastic and Reconstructive Surgery, Korea University Anam Hospital, 73, Inchon-ro, \\ Seongbuk-gu, Seoul 02841, Republic of Korea. Email: yesanam2@korea.ac.kr.
}

Background Fat grafting is now a common procedure for breast reconstruction. Many clinical studies have reported its aesthetic efficacy and oncological safety, but some experimental studies raise about the recurrence risk because of its regenerating property. This study aims to investigate the possibility of cancer recurrence associated with fat grafting.

Methods: In this retrospective cohort study, we analyzed a total of 339 patients who had undergone immediate reconstructive surgery after nipple-sparing mastectomy (NSM) or skin-sparing mastectomy (SSM) in our institution between February 28, 2009 and March 23, 2019. Patients who had undergone breast conserving surgery, radical mastectomy, or delayed reconstruction were excluded. We used univariate and multivariate Cox proportional hazards regression models to evaluate the association between fat grafting and cancer recurrence.

Results: Among the 339 patients during a median follow-up of 52 months, 27 patients (8.0\%) were confirmed to have recurrent cancer. Of 67 patients who had undergone fat grafting, 10 patients were confirmed to have cancer recurrence. In multivariate analyses, fat grafting [hazard ratio (HR), 2.52; 95\% CI, 1.005-6.317; $\mathrm{P}=0.0488$ ] was independently associated with cancer recurrence.

Conclusions: In population of breast cancer patient who underwent immediate reconstruction in our institution, fat grafting showed significant higher risk of cancer recurrence. Although these results are at odds with many existing studies, it suggests that more careful follow-up may be necessary for patients who had undergone fat grafting after reconstructive surgery.

Keywords: Fat graft; breast cancer; cancer recurrence; breast reconstruction

Submitted Jul 31, 2020. Accepted for publication Nov 13, 2020.

doi: $10.21037 /$ gs-20-645

View this article at: http://dx.doi.org/10.21037/gs-20-645

\footnotetext{
^ Jae-Ho Chung, ORCID: 0000-0002-8351-2444; Ki-Jae Kim, ORCID: 0000-0002-0979-6688; Seung Pil Jung, ORCID: 0000-0003-39672974; Seung-Ha Park, ORCID: 0000-0002-2667-2791; Eul-Sik Yoon, ORCID: 0000-0001-5734-6625.
} 


\section{Introduction}

Autologous fat grafting is now a common procedure for breast reconstruction. Many plastic surgeons often do fat transfer after reconstructive surgery to optimize the aesthetic outcome, and more recently, fat grafting with stem/progenitor cells extracted from the stromal vascular fraction (SVF) has been attempted to improve its characteristics and long term persistence (1). Also, several clinical studies have been published to demonstrate its aesthetic efficacy and oncological safety (2-4).

However, some surgeons are still concerned about the theoretical risk of cancer development by the stimulation of lipoaspirates grafts. Adipocytes could express protumorigenic factors, and stem cells could transform within the graft (5). In addition, the intentional placement of these regenerating tissues at close to a previous tumor bed raises questions about the possibility of promoting a cancer recurrence (6). Over the past decade, many basic studies have shown that fat grafting could stimulate cancer growth and proliferation (7-10).

This conflicting result between clinical and fundamental studies still leaves doubt about the safety of fat transfer or SVF enrichment in breast reconstruction. In recent years, several matched cohort studies have been published, however, most of them included heterogeneous populations and analyzed the outcome with different statistical methods. Therefore, in this clinical study, we analyzed the incidence of cancer recurrence associated with fat grafting in a patient who had undergone immediate reconstructive surgery after nipple-sparing mastectomy (NSM) or skin-sparing mastectomy (SSM) in our institution. The primary objective was to analyze whether fat grafting as an adjunct procedure increases the rate of cancer recurrence. The secondary objective was to investigate the safety of SVF enrichment in terms of cancer recurrence. We present the following article in accordance with the STROBE reporting checklist (available at http://dx.doi.org/10.21037/gs-20-645).

\section{Methods}

\section{Patients}

This retrospective analysis was approved by the Institutional Review Board of Korea University Anam Hospital (protocol number 2020AN0106) and performed in accordance with the principles of the Declaration of Helsinki (as revised in 2013). Individual consent for this retrospective analysis was waived. Medical records including detailed operation notes, follow-up records, and photographs were collected and analyzed. Patients who had undergone breast conserving surgery or radical mastectomy were excluded because the cohort was too small. Also, patients who had had a delayed reconstruction were excluded to achieve standardization. Finally, data for 339 patient who had received primary reconstructive surgery after NSM or SSM between February 28, 2009 and March 23, 2019 were included.

\section{Study design}

In our center, patients treated for breast cancer were regularly followed up every 6 months with mammography and ultrasonography for the 5 years and annually thereafter, depending on the primary pathological condition. Other than that, no additional imaging workup was performed, specifically before fat grafting. The cancer recurrence including loco-regional recurrence or distant metastasis was the primary end point of this study. If there were no events, the observation was censored at the last follow-up visit. To assess the effect of fat grafting on the risk of cancer recurrence, we compared outcomes between the fat grafting group $(n=67)$ and no lipofilling group $(n=272)$. Also, in the fat grafting group, patients who had undergone SVFenriched fat grafting $(\mathrm{n}=11)$ were compared with patients who had received lipofilling only $(\mathrm{n}=56)$ to assess the risk of SVF grafting.

\section{Fat grafting and SVF isolation technique}

In most cases, a fat transfer was performed under local anesthesia at least 6 months after tumor resection except in cases that general anesthesia was required for implant change in two-stage reconstruction. The median interval from tumor resection to fat graft was 8 months (range, 3-99 months).

Tumescence included $1 \mathrm{~L}$ of normal saline with $20-\mathrm{mL}$ of lidocaine and $1 \mathrm{~mL}$ of 1:1,000 epinephrine was injected evenly into the donor site, such as the lower abdomen, flank or thigh. Harvesting was performed conventionally with a 3-mm Coleman suction tube (Coleman cannula, Byron Medical, Tucson, AZ, USA), and a 10-mL syringe (Coleman system, Byron Medical, Tuscon, AZ, USA). Then fat was centrifuged at 3,000 rpm for $3 \mathrm{~min}$ to obtain purified fat. After that, purified fat was transferred to a $10-\mathrm{mL}$ syringe for placement into the target region.

In our study, a total of 11 patients had undergone SVFenriched fat grafting. SVFs were isolated from harvested 
fat using the Smart X kit (Dongkoo Bio \& Pharm, Seoul, South Korea) according to the manufacturer's instructions. Because $10-\mathrm{mL}$ of fat is usually extracted as much as $1 \mathrm{cc}$ of SVF, in patients planned the SVF grafting, a larger amount of fat was harvested in consideration.

\section{Statistical analyses ${ }^{1}$}

In patient demographic analysis, categorical variables were expressed as counts (percentages) with Chi-squared tests or Fisher's exact tests. Continuous variables were summarized by means (standard deveiations) or medians (interquartile ranges) with two independent $t$-tests or Mann-Whitney tests depending on whether normality was satisfied.

Univariate and multivariable Cox's proportional hazards regression models were used to analyze the effects of the fat grafting group compared to the control group for cancer recurrence after adjusting for clinicopathologic variables. The variables such as fat graft, age, BMI, comorbidity (hypertension, diabetes), smoking, cancer type, tumor size, tumor quadrant, grade, calcification, pathological staging, hormone receptor status (ER, PR, HER2/neu, Ki-67), mastectomy type, reconstructive type, chemotherapy and radiotherapy were included in multivariable Cox's proportional hazard regression model. The final model with some important factors was obtained by stepwise variable selection method. The proportional hazards assumption was checked using Supremum test and graphical diagnostics based on the scaled Schoenfeld residuals. Meanwhile, we also performed the same statistical analysis to find the effects of SVF on cancer recurrence in the only fat grafting group. For all analyses, a value of $\mathrm{P}<0.05$ was considered statistically significant. All statistical analyses were performed using IBM SPSS Statistics, version 22.0 (IBM Corp., Armonk, NY) and SAS, version 9.4 (SAS Institute Inc., Cary, NC).

\section{Results}

\section{Patient demographics}

We analyzed a total of 339 patients including 143 nipplespraing mastectomies and 196 skin-sparing mastectomies. Among the 339 patients, a total of 67 patients received fat grafting, of which 11 patients received SVF-enriched fat grafting. Most of the reconstruction procedures used a pedicled latissimus dorsi flap $(\mathrm{n}=108,31.9 \%)$ and two-stage implant reconstruction $(\mathrm{n}=160,47.2 \%)$. Of the 339 patients, $192(56.6 \%)$ received chemotherapy and $36(10.6 \%)$, adjuvant radiotherapy. Table 1 summarizes the patient demographics and clinical characteristics.

Table 2 summarizes the characteristics of the fat grafting

Table 1 Patient demographics

\begin{tabular}{|c|c|c|c|c|c|c|}
\hline Variable & \multicolumn{2}{|c|}{ Fat grafting } & \multicolumn{2}{|c|}{ Controls } & Total & $\mathrm{P}^{\dagger}$ \\
\hline No. of patients & 36 & 31 & 114 & 158 & 339 & \\
\hline Recurrence, n (\%) & $5(13.9)$ & $5(16.1)$ & $9(7.9)$ & $8(5.1)$ & $27(8.0)$ & $0.026^{*}$ \\
\hline Mean age, years & 51.2 & 47.0 & 52.0 & 49.8 & 50.4 & 0.210 \\
\hline Smoking, n (\%) & $1(2.8)$ & $1(3.2)$ & $8(7.0)$ & $7(4.4)$ & $17(5.0)$ & 0.210 \\
\hline \multicolumn{7}{|l|}{ Comorbidity, n (\%) } \\
\hline Hypertension & $2(5.6)$ & $2(6.4)$ & $13(11.4)$ & $17(10.8)$ & $34(10.0)$ & 0.306 \\
\hline Diabetes & $1(2.8)$ & 0 & $3(2.6)$ & $7(4.4)$ & $11(3.2)$ & 0.733 \\
\hline
\end{tabular}

Table 1 (continued)

\footnotetext{
${ }^{1}$ All statistical analyses were performed in consultation with an independent medical statistician.
} 
Table 1 (continued)

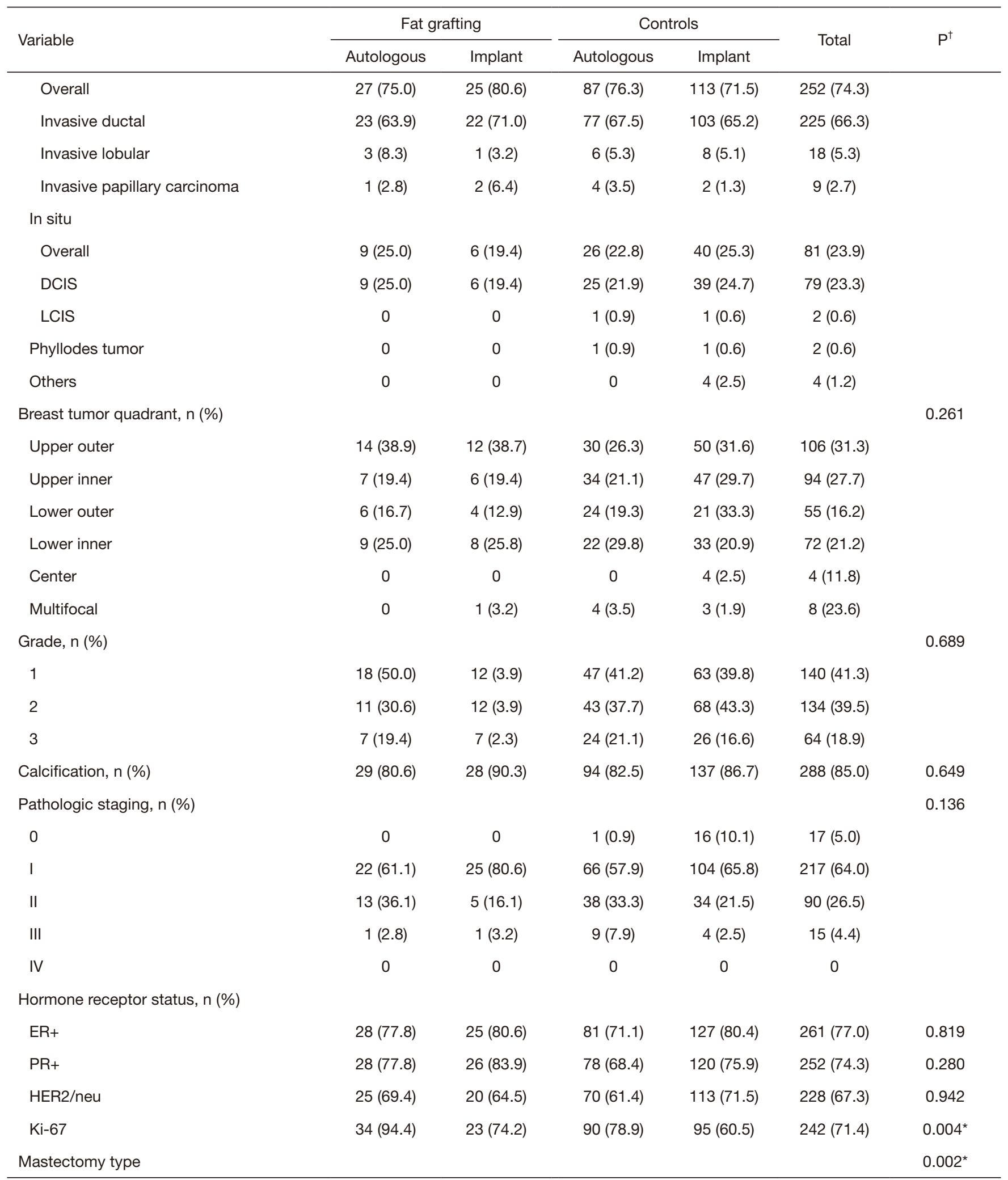

Table 1 (continued) 
Table 1 (continued)

\begin{tabular}{|c|c|c|c|c|c|c|}
\hline Variable & \multicolumn{2}{|c|}{ Fat grafting } & \multicolumn{2}{|c|}{ Controls } & Total & $\mathrm{P}^{\dagger}$ \\
\hline NSM & $9(25.0)$ & $9(29.0)$ & $45(39.5)$ & $80(50.6)$ & $143(42.2)$ & \\
\hline SSM & $27(75.0)$ & $22(72.0)$ & $69(60.5)$ & $78(49.4)$ & $196(57.8)$ & \\
\hline Reconstructive type, n (\%) & & & & & & 0.148 \\
\hline TRAM & $6(16.7)$ & N/A & $19(16.7)$ & N/A & $25(7.4)$ & \\
\hline DIEP & $1(2.8)$ & $\mathrm{N} / \mathrm{A}$ & $16(14.0)$ & $\mathrm{N} / \mathrm{A}$ & $17(5.0)$ & \\
\hline LD & $29(80.6)$ & N/A & 79 (69.3) & N/A & $108(31.9)$ & \\
\hline \multicolumn{7}{|l|}{ Implant } \\
\hline Chemotherapy & & & & & & 0.658 \\
\hline Overall & $17(47.2)$ & $17(54.8)$ & $70(61.4)$ & $88(55.7)$ & $192(56.6)$ & \\
\hline Neoadjuvant & $1(2.8)$ & $4(12.9)$ & $11(9.6)$ & $17(10.8)$ & $33(9.7)$ & \\
\hline Adjuvant & $16(44.4)$ & $13(41.9)$ & 59 (51.8) & $71(44.9)$ & $159(46.9)$ & \\
\hline Radiation therapy & $6(16.7)$ & $6(19.4)$ & $14(12.3)$ & $10(6.3)$ & $36(10.6)$ & $0.025^{*}$ \\
\hline
\end{tabular}

${ }^{\dagger}$, above $\mathrm{P}$ value was calculated by chi-square test or Fischer exact test or $t$-test or Mann-Whitney test for the difference between fat grafting group and control group; *, statistically significant. Patients underwent LD flap with implant insertion were classified to autologous flap group. No., number; yr, years; BMI, body mass index; DCIS, ductal carcinoma in situ; LCIS, lobular carcinoma in situ; ER, estrogen receptor; PR, progesterone receptor; HER2, human epidermal growth factor receptor 2; Ki-67, Ki-67 protein is a cellular marker for proliferation; NSM, nipple sparing mastectomy; SSM, skin sparing mastectomy; TRAM, transverse rectus abdominis myocutaneous flap; DIEP, deep inferior epigastric perforator flap; LD, latissimus dorsi.

Table 2 Characteristic of patient underwent fat grafting

\begin{tabular}{lc}
\hline Variable & No. (\%) \\
\hline No. of patients & 67 \\
No. of sessions & $59(88.1)$ \\
1 & $7(10.4)$ \\
2 & $1(1.5)$ \\
$\geq 3$ & \\
Total volume injected & $51(76.1)$ \\
$1-100$ cc & $15(22.3)$ \\
$101-200$ cc & $1(1.5)$ \\
$201-300$ cc & $11(16.4)$ \\
No. of SVF graft &
\end{tabular}

group. In this study, patients had undergone an average of 1.1 lipofilling sessions (range, $1-3)$ and most of them ( $n=59$, $88.1 \%)$ required just one lipofilling procedure. The amount of fat injected was ranged from 20 to $224 \mathrm{cc}$, with a mean of 83 cc. Of 67 patients, 11 patients received SVF-enriched fat grafting. The mean volume of injected SVF was $5.7 \mathrm{cc}$.

\section{Cancer recurrence}

In a median follow-up duration of 52 months (range, 15-120 months), 27 patients $(8.0 \%)$ were confirmed to have recur breast cancer as a loco-regional recurrence $(n=25)$, regional lymph node recurrence $(n=1)$ or distant metastasis $(n=1)$. The characteristics and outcomes of the 27 patients who developed cancer recurrence are shown in Table 3. These patients had a median age of 50 years 


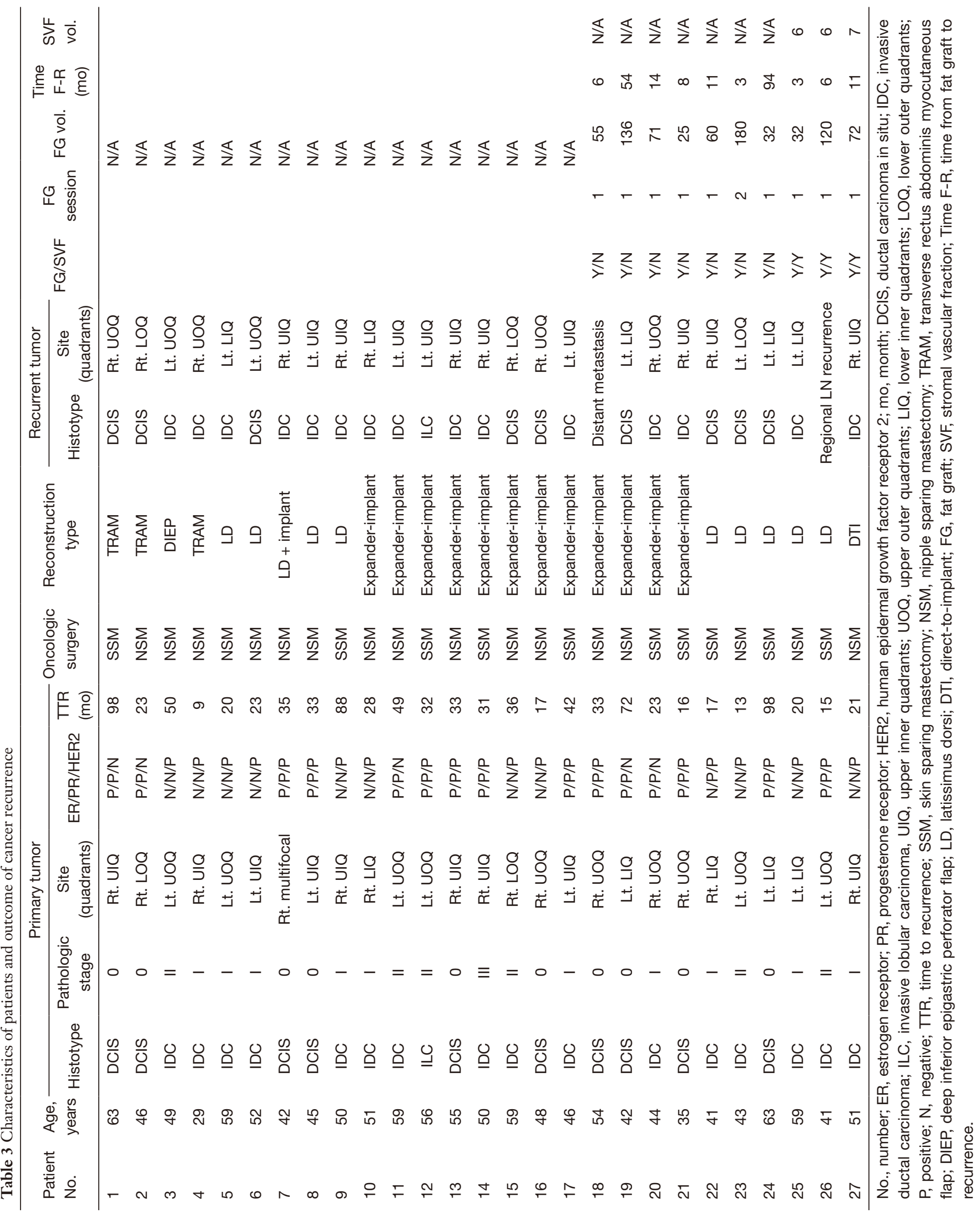


Table 4 Multivariable Cox's proportional hazard survival model using stepwise selection

\begin{tabular}{lccc}
\hline & Hazard ratio & $95 \%$ confidence limits & P value \\
\hline Fat graft (+) vs. Fat graft (-) & 2.52 & $1.005-6.317$ & 0.0488 \\
Calcification (+) vs. Calcification (-) & 2.976 & $1.190-7.407$ & 0.0196 \\
HER2/neu (+) vs. HER2/neu (-) & 3.502 & $1.179-10.407$ & 0.0241 \\
NSM vs. SSM & 3.817 & $1.502-9.7$ & 0.0049 \\
\hline
\end{tabular}

We used multivariable cox's hazard survival model for statistical analysis. Variables including fat graft, age, BMI, comorbidity (hypertension, diabetes), smoking, cancer type, tumor quadrant, grade, calcification, pathologic staging, hormone receptor status (ER, PR, HER2/neu, $\mathrm{Ki}-67)$, mastectomy type, reconstructive type, chemotherapy, radiotherapy were fitted to this model, and stepwise selection was performed. HER2, human epidermal growth factor receptor 2; NSM, nipple sparing mastectomy; SSM, skin sparing mastectomy.

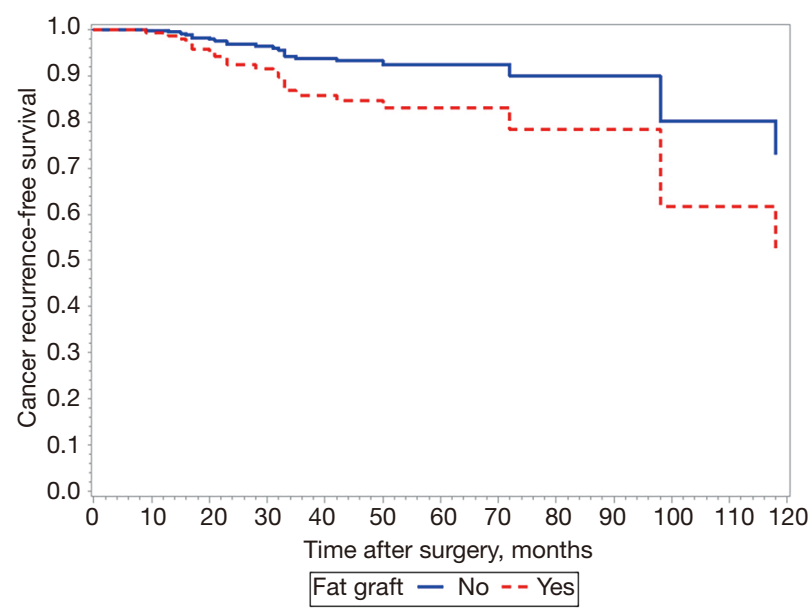

Figure 1 Survival plot obtained by multivariable Cox's proportional hazard model.

(range, 29-63 years). The histotypes of the primary tumor were invasive ductal carcinoma in 16 cases (59.3\%), ductal carcinoma in situ in 10 cases $(37.0 \%)$ and invasive lobular carcinoma in 1 case $(3.7 \%)$. The histotypes of recurrent tumor were invasive ductal carcinoma in 15 cases $(55.6 \%)$, ductal carcinoma in situ in 9 cases $(33.3 \%)$ and invasive lobular carcinoma in 1 case $(3.7 \%)$. The other two patients were confirmed as having regional lymph node recurrence and distant metastasis, respectively. The median time from surgery to cancer recurrence was 31 months (range, 9-98 months). Of the 67 patients who had undergone fat grafting, 10 patients were confirmed as having cancer recurrence. The median time from fat grafting to cancer recurrence 9.5 months (range, 3-94 months).

\section{Risk factors for cancer recurrence}

We used a multivariable Cox's hazard survival model for statistical analysis including the time variable. Table 4 summarizes the selected variables with statistical significance using stepwise selection. Of the above mentioned variables, fat graft (HR 2.52; 95\% CI, 1.005-6.317; P=0.0488), calcification (HR 2.976; 95\% CI, 1.190-7.407; P=0.0196), HER2/neu-positive subtype (HR 3.502; 95\% CI, 1.17910.407; $\mathrm{P}=0.0241$ ) and NSM (HR 3.817; 95\% CI, 1.502-9.7, $\mathrm{P}=0.0049)$ were independently associated with cancer recurrence. Figure 1 shows a survival plot by fat grafting obtained after adjusting other variable.

To analyze the risk of SVF-enriched fat grafting, univariate cox's hazard regression was performed (Table 5). The SVF graft shows an increased hazard ratio (HR 2.916; 95\% CI, 0.564-15.074), although it is not statistically significant $(\mathrm{P}=0.202)$. Figure 2 shows a survival plot by SVF graft obtained by this univariate Cox's proportional hazard model.

\section{Discussion}

Autologous fat grafting is a popular choice for surgeons to correct the deformity after total or partial breast reconstruction. However, there remains no consensus about the oncological safety of fat grafting. Although some studies have demonstrated the possibility of tumorigenicity by fat grafting in 'in vitro' and in animal models (8-10), but in contrast, opposing data have been published, showing that fat grafting may inhibit tumor growth and metastasis $(11,12)$.

In the literature, five matched case-control studies have been published that did not reveal any increasing risk of local recurrences in their fat grafting group. First, Rigotti et al. reported the outcome of 137 patients who had undergone fat tissue transplant after modified radical mastectomy for a median follow-up of 7.6 years (13). They compared the risk of local recurrence between mastectomy and fat grafting to the risk observed in the period after 
Table 5 Univariate Cox's proportional hazard regression after SVF-enriched fat graft

\begin{tabular}{lccc}
\hline & Hazard ratio & 95\% confidence limits & P value \\
\hline SVF graft (+) vs. SVF graft (-) & 2.916 & $0.564-15.074$ & 0.2017 \\
\hline
\end{tabular}

SVF, stromal vascular fraction.

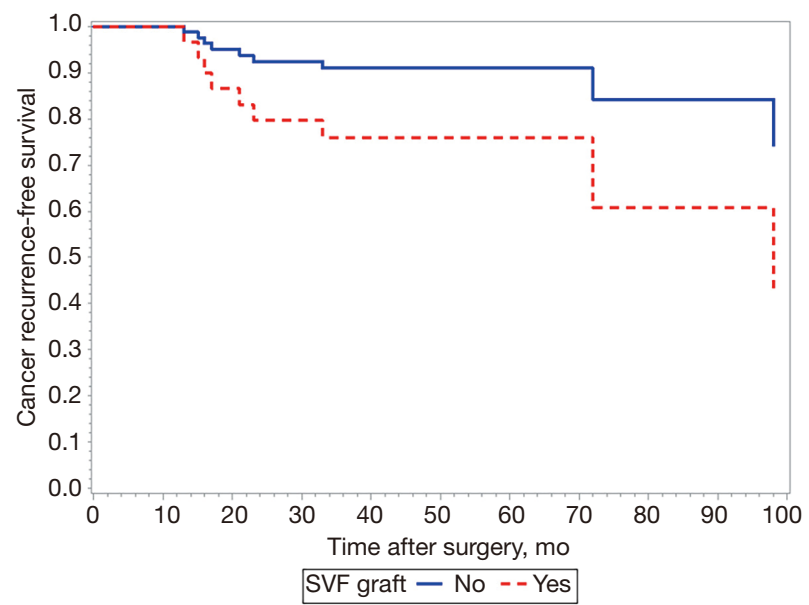

Figure 2 Survival plot obtained by univariate Cox's proportional hazard model.

fat grafting, and concluded that lipoaspirate in breast reconstruction does not increase the incidence of local recurrence of breast cancer. Second, Petit et al. published two articles in 2012 and 2017 (14,15). In their first study conducted at the European Institute of Oncology (IEO), there was no statistical difference in the local recurrence rates between 321 patients with breast cancer who had received fat grafting and a matched control group of 642 patients (HR 1.1; 95\% CI, 0.47-2.64; $\mathrm{P}=0.79$ ). In their second study, they retrospectively compared 322 patients receiving breast conserving surgery followed by fat grafting and 322 matched patients with similar characteristics who did not undergo fat grafting. After a mean follow-up of 4.6 years after fat grafting, they observed no difference in the incidence of local events $(\mathrm{P}=0.49)$, axillary nodes metastasis $(\mathrm{P}=0.23)$, distant metastasis $(\mathrm{P}=0.67)$ or contralateral breast $(\mathrm{P}=0.51)$. Fourth, Gale et al. published their study using a protocol similar to Petit's (16). Their study gathered 211 lipofilling cases composed of 176 mastectomies and 35 breast conserving surgeries, and compared with a matched control group of 422 patients. For a mean follow-up of 88 months, they found no evidence of increased oncological risk associated with fat grafting $(\mathrm{P}=0.74)$. Finally, Kronowitz et al. reported that 719 patients who had undergone segmental or total mastectomy for breast cancer followed by fat grafting showed no increase in locoregional recurrence or systemic recurrence compared with 670 patients had undergone reconstruction without lipofilling (17).

However, there are some limitations in interpreting these individual studies reported in the literature. They are heterogenous and some include cosmetic breast patients without exposure to breast cancer (16). Also, several studies analyzed the outcome using Kaplan-Meier method, however, it did not adjust many confounding variables. In contrast, the multivariate Cox's proportional hazard regression model allows analysis of the effects of various variables on the occurrence of a particular event. In this respect, Kronowitz's research provides a more favorable result, because it included more patients and more adequate statistical method. However, their study included all patients who had undergone segmental mastectomy or total mastectomy as subjects and it is not clear whether they evaluated the effects of reconstructive surgery such as autologous flap or implant-based reconstruction. From this point of view, our study is different, in that the scope of the study was limited to patients who had undergone fat grafting followed by immediate reconstructive surgery after NSM or SSM.

So far, many experimental studies have reported data about the possibility of endocrine, paracrine and autocrine activity of the transplanted fat tissue. Most of them were concerned that the intentional placement of these regenerating tissues at close to a previous tumor bed could be related with promoting a cancer recurrence. Wang et al. reported that cancer-associated adipocytes, referring tumor-surrounding adipocytes, appear to promote tumor progression by acting directly on the target tumor cell (18). Also, Lohsiriwat et al. pointed out that "the tumor-stroma interaction" can potentially induce cancer reappearance by fueling dormant breast cancer cells in the tumor bed (12). At least in these basic experimental studies, the possibility of relationship between fat grafting and cancer recurrence is well established.

In our study, patients who had undergone fat grafting showed a higher hazard ratio (HR 2.52; $95 \%$ CI, $1.005-6.317)$ with statistical significance $(\mathrm{P}=0.0488)$ by 
multivariable Cox's hazard regression model. Because these findings are at odds with several other studies in the literature as cited in above, we thought it might have reflected some differences in patient demographics between two cohorts. In this study, a significantly higher percentage of patients in the fat grafting cohort underwent NSM $(\mathrm{P}=0.002)$ and radiated therapy $(\mathrm{P}=0.025)$. Considering that NSM was independently associated with cancer recurrence in stepwise selection of Cox's hazard survival model (HR 3.817 ; 95\% CI, $1.502-9.7, \mathrm{P}=0.0049$ ), it seems that this difference in patient composition may have resulted in different results from previous studies. From the result of this study, we concluded that it should be kept with intervals of at least 6 months for the interval between tumor resection and fat graft and more careful follow-up may be necessary.

Meanwhile, Myckatyn et al. recently reported a multicenter case-cohort study with regard to this subject (19). They concluded that fat transfer was not associated with an increased probability of cancer recurrence in patients with stage I through III invasive ductal carcinomas. There is a difference in that their study focused on patients with invasive ductal carcinoma only, whereas we included all the types of cancers such as ductal carcinoma in situ, lobular carcinoma in situ and the other types of invasive carcinoma. However, it was very impressive in that it overcame the shortcoming of previous studies, by multi-institutional case cohort design with large samples. We expect that more clinical studies in multicenter could clearly conclude the issue of fat grafting on cancer recurrence.

In this study, we included 11 patients who had undergone SVF-enriched fat grafts. There are differences between the two procedures: the simple purification of the liposuction specimen and the enrichment of stromal vascular fraction (SVF). The first, so-called Coleman technique, does not modify the concentration of SVF. However, the second, the so-called enrichment technique, increased the concentration of SVFs in the specimen that will be used for the reconstruction (20).

In the literature, there are few studies about the safety of SVF-enrichment lipofilling in breast cancer. First, PérezCano et al. introduced their prospective trial of adiposederived regenerative cell (ADRC)-enriched fat grafting for partial mastectomy defects, called the RESTORE-2 trial (21). Of the 67 patients treated in this study, 50 reported satisfaction and no reported local cancer recurrence during 12 months follow-up. Second, Mazur et al. reported that here was no statistical difference in the local recurrence rates between 56 patients with breast cancer who had received SVF-enriched fat grafting (3.7\%) and a matched control group of 252 patients (4.13\%) for a 3 -year-observation time (22). However, they stated that their results need a cautious interpretation, because the cohort of this study shows heterogeneity of both cancer type and cancer treatment protocol. Last, Calabrese et al. performed prospective study of 169 patients who had undergone twostage breast reconstruction after NSM to evaluate the longterm cancer recurrence risk of SVF enriched fat grafting (23). In their study, the SVF enrichment group showed a 1.92 odds ratio (95\% CI, 0.47-10.4) in comparison to patients who did not undergo fat grafting; however, there was no significant difference in regression analysis $(\mathrm{P}=0.447)$.

In our study, the SVF enrichment group showed a 2.92 hazard ratio (95\% CI, 0.564-15.074) without statistically significance $(\mathrm{P}=0.202)$. If more patients who had received SVF-enriched fat grafting were included in this study, however, other significant results may have been obtained. Krumboeck et al. pointed out that there are no data about a threshold for the stem cell rate that can be used safely during fat grafting at present (24). Laloze et al. also carefully recommend that further studies with long-term followup are needed to assess the risk of cancer and complication with cell-assisted lipotransfer in their systematic review and meta-analysis (25). We agreed with their recommendations that we needs more scientific data and prospectively controlled long-term clinical studies. Therefore, it will be necessary to explain the gain and loss to the patients and receive the informed consent about SVF-enriched fat grafting.

Our study has several limitations. First, the retrospective design of this study could include bias, and it did not use a perfectly matched cohort. The matched case-control design, which matches each case, will make a result more reliable in this type of study with heterogeneous population. Second, it included a relatively few patients, using only single-center data. As mentioned above, the ideal design of this type of study may be multicenter randomized clinical trials with more long-term follow-up. Last, to verify the safety of SVFenriched fat grafting, more data are needed. Also, future studies should include patients who underwent fat grafting after delayed reconstruction and after breast conservation surgery (19).

\section{Conclusions}

To the best of our knowledge, this article is the first clinical 
comparative study reporting the significant association between fat grafting and cancer recurrence. Although this result seems to reflect difference in patient composition in two cohorts, it reminds that more careful follow-up may be necessary in patients underwent fat grafting after reconstructive surgery.

Meanwhile, SVF-enriched fat grafting does not show a significant relationship with cancer recurrence in our study, however, we analyzed a limited number of patients. Therefore, more data are needed about SVF-enriched fat grafting to analyze the effect of stem cells on cancer recurrence at breast level and establish a threshold for the stem-cell rate, which can be used safely during fat grafting.

\section{Acknowledgments}

The first author (JHC) expressed his gratitude for the aid of Kyung-Sook Yang, a professor affiliated with the Department of Biostatistics in Korea University College of Medicine. She supervised all statistical analysis done by the first author (JHC).

Funding: None.

\section{Footnote}

Reporting Checklist: The authors have completed the STROBE reporting checklist. Available at http://dx.doi. org/10.21037/gs-20-645

Data Sharing Statement: Available at http://dx.doi. org/10.21037/gs-20-645

Conflicts of Interest: All authors have completed the ICMJE uniform disclosure form (available at http://dx.doi. org/10.21037/gs-20-645). The authors have no conflicts of interest to declare.

Ethical Statement: The authors are accountable for all aspects of the work in ensuring that questions related to the accuracy or integrity of any part of the work are appropriately investigated and resolved. The study was conducted in accordance with the Declaration of Helsinki (as revised in 2013). The study was approved by the institutional review board of Korea University Anam Hospital (protocol number 2020AN0106). Individual consent for this retrospective analysis was waived.

Open Access Statement: This is an Open Access article distributed in accordance with the Creative Commons Attribution-NonCommercial-NoDerivs 4.0 International License (CC BY-NC-ND 4.0), which permits the noncommercial replication and distribution of the article with the strict proviso that no changes or edits are made and the original work is properly cited (including links to both the formal publication through the relevant DOI and the license). See: https://creativecommons.org/licenses/by-nc-nd/4.0/.

\section{References}

1. Bielli A, Scioli MG, Gentile P, et al. Adipose TissueDerived Stem Cell Therapy for Post-Surgical Breast Reconstruction: More Light than Shadows. Adv Clin Exp Med 2015;24:545-8.

2. Riggio E, Bordoni D, Nava MB. Oncologic surveillance of breast cancer patients after lipofilling. Aesthetic Plast Surg 2013;37:728-35.

3. Stumpf CC, Blazus JV, Zucatto FSAE, et al. Immediate reconstruction with autologous fat grafting: Influence in breast cancer regional recurrence. Rev Col Bras Cir 2017;44:179-86.

4. Silva-Vergara C, Fontdevlla J, Descarrega J, et al. Oncological outcomes of lipofilling breast reconstruction: 195 consecutive cases and literature review. J Plast Reconstr Aesthet Surg 2016;69:475-81.

5. Hefetz-Sela S, Schrer PE. Adipocytes: Impact on tumor growth and potential sites for therapeutic intervention. Pharmacol Ther 2013;138:197-210.

6. Krastev TK, Jonasse Y, Kon M. Onoclogical safety of autologous lipoaspirate grafting in breast cancer patients: a systematic review. Ann Surg Oncol 2013;20:111-9.

7. Orbay H, Hinchcliff KM, Charvet HJ, et al. Fat Graft Safety after Oncologic Surgery: Addressing the Contradiction between In Vitro and Clinical Studies. Plast Reconstr Surg 2018;142:1489-99.

8. Zhang Y, Daguinag A, Traktuev DO, et al. White adipose tissue cells are recruited by experimental tumors and promote cancer progression in mouse models. Cancer Res 2009;69:5259-66.

9. Yu JM, Jun ES, Jung JS, et al. Mesenchymal stem cells derived from human adipose tissues favor tumor cell growth in vivo. Stem Cells Dev 2008;17:463-73.

10. Muehlberg FL, Song YH, Krohn A, et al. Tissue-resident stem cells promote breast cancer growth and metastasis. Carcinogenesis 2009;30:589-97.

11. Dirat B, Bochet L, Dabek M, et al. Cancer-associated adipocytes exhibit an activated phenotype and contribute 
to breast cancer invasion. Cancer Res 2011;71:2455-65.

12. Lohsiriwat V, Curigilano G, Rietjens M, et al. Autologous fat transplantation in patients with breast cancer:

"silencing" or "fueling" cancer recurrence? Breast 2011;20:351-7.

13. Rigotti G, Marchi A, Stringhini P, et al. Determining the oncological risk of autologous lipoaspirategrafting for post-mastectomy breast reconstruction. Aesthetic Plast Surg 2010;34:475-80.

14. Petit JY, Botteri E, Lohsiriwat V, et al. Locoregional recurrence risk after lipofilling in breast cancer patients. Ann Oncol 2012;23:582-8.

15. Petit JY, Maisonneuve P, Rotmensz N, et al. Fat Grafting after Invasive Breast Cancer: A Matched Case-Control Study. Plast Reconstr Surg 2017;139:1292-6.

16. Gale KL, Rakha EA, Ball G, et al. A case-controlled study of the oncologic safety of fat grafting. Plast Reconstr Surg 2015;135:1263-75.

17. Kronowitz SJ, Mandujano CC, Liu J, et al. Lipofilling of the Breast Does Not Increase the Risk of Recurrence of Breast Cancer: A Matched Controlled Study. Plast Reconstr Surg 2016;137:385-93.

18. Wang YY, Lehuede C, Laurent V, et al. Adipose tissue and breast epithelial cells: a dangerous dynamic duo in breast cancer. Cancer Lett 2012;324:142-51.

19. Myckatyn TM, Wagner IJ, Mehrara BJ, et al. Cancer Risk

Cite this article as: Chung JH, Kim KJ, Jung SP, Park SH, Yoon ES. Analysis of oncological safety of autologous fat grafting after immediate breast reconstruction. Gland Surg 2021;10(2):584-594. doi: 10.21037/gs-20-645 after Fat Transfer: A Multicenter Case-Cohort Study. Plast Reconstr Surg 2017;139:11-8.

20. Petit JY, Maisonneuve P, Rotmensz N, et al. Safety of Lipofilling in Patients with Breast Cancer. Clin Plast Surg 2015;42:339-44.

21. Pérez-Cano R, Vranckx JJ, Lasso JM, et al. Prospective trial of adipose-derived regenerative cell (ADRC)enriched fat grafting for partial mastectomy defects: The RESTORE-2 trial. Eur J Surg Oncol 2012;38:382-9.

22. Mazur S, Zolocinska A, Pojda Z, et al. Safety of adiposederived cell (SVF) augmentation for surgical breast reconstruction in cancer patients. Adv Clin Exp Med 2018;27:1085-90.

23. Calabrese C, Kothari A, Badylak S, et al. Oncological safety of stromal vascular fraction enriched fat grafting in two-stage breast reconstruction after nipple sparing mastectomy: Long-term results of a prospective study. Eur Rev Med Pharmacol Sci 2018;22:4768-77.

24. Krumboeck A, Giovanoli P, Plock JA. Fat grafting and stem cell enhanced fat grafting to the breast under oncological aspects: recommendations for patient selection. Breast 2013;22:579-84.

25. Laloze J, Varin A, Gilhodes J, et al. Cell-assisted lipotransfer: Friend or foe in fat grafting? Systematic review and meta-analysis. J Tissue Eng Regen Med 2018;12:e1237-e1250. 